\title{
A Volumetric Optical Flow Method for Measurement of Brain Deformation from Intraoperative Magnetic Resonance Images
}

\author{
Nobuhiko Hata, Arya Nabavi, Simon Warfield, William Wells, \\ Ron Kikinis, and Ferenc A. Jolesz \\ Image-guided Therapy Program, Department of Radiology \\ Brigham and Women's Hospital and Harvard Medical School \\ 75 Francis St., Boston, MA 02115, USA \\ \{noby,navabi,warfield,sw,kikinis,jolesz\}@ bwh.harvard.edu
}

\begin{abstract}
A method to measure spatial and temporal brain deformation from sequential intraoperative Magnetic Resonance Images (MRI) and its preliminary clinical results are reported. Deformation is estimated with a volumetric optical flow measurement based on local intensity differences. A multi-resolution approach was used to efficiently estimate the deformation. We applied the method to five different cases and the method is highlighted by illustrative features accompanied by five sets of intraoperative MRI scanned before and after dura opening, twice during tumor resection and immediately after dura closure. The maximum cortical surface shift measured was $11 \mathrm{~mm}$ and subsurface shift was $4 \mathrm{~mm}$. Volume change was measured by aligning the sequence of intraoperative MR images immediately after the opening of the dura to the images during the tumor resection. The amount of deformation present at each stage of the surgery was visualized. The computed deformation field was most satisfactory when the skin was first segmented and removed from the images prior to the optical flow computation. Magnetic field inhomegeneities as well as administration of contrast agent (GadoliniumDTPA) were observed to modify the deformation field. The method demonstrated a good capability of intra-operative surface, subsurface and midline shift measurement.
\end{abstract}

\section{Introduction}

The evolution of image-guided neurosurgery raises intraoperative brain shift as a problematic issue, since any spatial discrepancy between coregistered preoperative image and the shifted brain diminishes the accuracy of neuronaviagtion. Kato and Koivukangus $[1,2]$ pointed out, from their clinical experience with a neuronavigation system, that significant brain shift is observed after cerebrospinal fluid is drained and during tumor resection.

Ideally, more accurate and reliable image-guided-navigation, the preoperative image should be updated and deformed to reflect intraoperative shape change of the brain. This can be accomplished by performing deformable registration of pre- to intra-operative images. 
This paper will introduce our approach to assess intra-cranial structural change by optical flow measurement. To gain true intraoperative observation of brain deformations, we performed brain surgeries in an intraoperative Magnetic Resonance Imaging scanner (Signa SP, General Electric Medical Systems, Milwaukee, WI). The scanner has two toroidal magnetic coils and allows access to the surgical field through a 56-cm gap between the magnet coils.

\section{Methods}

We developed a method to measure brain deformation from intensity change in sequential MR-images. Deformation is estimated with a volumetric optical flow measurement based on local intensity differences. A multi-resolution approach was used to efficiently estimate the deformation. The method was originally developed for two-dimensional image measurement, and we modified it to process volumetric images.

Optical flow is a method for computing a motion field from images and has been employed mostly in the computer vision and artificial intelligence community since the late 1970's. By definition, optical flow is image velocity approximating image motion from sequential time-ordered images. Optical flow has been applied to motion detection, segmentation and motion-compensated image encoding. Detailed comparison of various optical flow methods appears in Barron and Bauchemin's report [3].

The underlying assumption of optical flow is that image intensity $E$ of a moving point $(x, y, z)$ at a time point $t$ is constant after a short duration of time $\delta t$. If a vector $(u, v, w)$ represents a velocity of the point and the intensity of the point doesn't change over the time $\delta t$, we can formulate,

$$
E(x+u \delta t, y+v \delta t, z+w \delta t, t+\delta t)=E(x, y, z, t)
$$

Assuming that the image intensity varies smoothly with $x, y, z$ and $t$, we can use a first-order Taylor expansion of the left hand side of (1):

$$
E(x, y, z, t)+u \delta t \frac{\partial E}{\partial x}+v \delta t \frac{\partial E}{\partial y}+w \delta t \frac{\partial E}{\partial z}+\delta t \frac{\partial E}{\partial t} \approx E(x, y, z, t)
$$

Dividing by $\delta t$, and abbreviating the first-order derivatives by using subscripts, we can obtain

$$
E_{x} u+E_{y} v+E_{z} w+E_{t}=0
$$

Equation (3) is the optical flow constraint equation to solve for $(u, v, w)$. However, as is in two-dimensional cases, we cannot solve $(u, v, w)$ in the direction of the isobrightness contours, or along intensity gradient (details in [4] "the aperture problem").

We followed the approach of Horn and Shunck [4] for solving underdetermined optical flow problem. We construct a total error measure $\mathrm{E}(\alpha)$ as the spatial integral of two terms; the first term $\mathrm{E}_{o}=\left(E_{x} u+E_{y} v+E_{z} w+E_{t}\right)^{2}$ is the square of the error from the optical flow constraint (3), and second term 
$\mathrm{E}_{s}=u_{x}^{2}+u_{y}^{2}+u_{z}^{2}+v_{x}^{2}+v_{y}^{2}+v_{z}^{2}+w_{x}^{2}+w_{y}^{2}+w_{z}^{2} \quad$ is $\quad$ a penalty that encourages smoothness:

$$
\mathrm{E}(\alpha)=\iiint\left(\mathrm{E}_{o}(x, y, z, t)+\alpha^{2} \mathrm{E}_{s}(x, y, z, t)\right) d x d y d z
$$

$\alpha^{2}$ is a relative weight of the two terms and determined by considering the signalto-noise ration of the second term $\mathrm{E}_{s}$.

The smoothness constraint $\mathrm{E}_{s}$ was derived from Horn and Shunck's smoothness constraint in the two-dimensional case [4]. The assumption here is that neighboring points on the objects have similar displacements. We may solve (4) by the calculus of variations [5]. The Euler-Lagrange equations yield:

$$
\begin{aligned}
& \nabla^{2} u=\frac{1}{\alpha^{2}}\left(E_{x} u+E_{y} v+E_{z} w+E_{t}\right) E_{x} \\
& \nabla^{2} v=\frac{1}{\alpha^{2}}\left(E_{x} u+E_{y} v+E_{z} w+E_{t}\right) E_{y} \\
& \nabla^{2} w=\frac{1}{\alpha^{2}}\left(E_{x} u+E_{y} v+E_{z} w+E_{t}\right) E_{z}
\end{aligned}
$$

where $\nabla^{2}$ is the Laplacian operator $\nabla^{2}=\frac{\partial^{2}}{\partial x^{2}}+\frac{\partial^{2}}{\partial y^{2}}+\frac{\partial^{2}}{\partial z^{2}}$.

We approximate the Laplacians of $u, v$ and $w$ by

$$
\nabla^{2} u \approx \bar{u}_{i, j, k}-u_{i, j, k}, \nabla^{2} v \approx \bar{v}_{i, j, k}-v_{i, j, k}, \nabla^{2} w \approx \bar{w}_{i, j, k}-w_{i, j, k}
$$

where $\bar{u}_{i, j, k}, \bar{v}_{i, j, k}$ and $\bar{w}_{i, j, k}$ denote Gaussian weighted average $(\sigma=0.75)$ of the neighbor points around the point at $(i, j, k)$.

Combining (5) with (6) and isolating $u, v$ and $w$ leads to

$$
\begin{aligned}
& u=\bar{u}-\frac{E_{x}\left(E_{x} \bar{u}+E_{y} \bar{v}+E_{z} \bar{w}+E_{t}\right)}{\alpha^{2}+E_{x}^{2}+E_{y}^{2}+E_{z}^{2}} \\
& v=\bar{v}-\frac{E_{y}\left(E_{x} \bar{u}+E_{y} \bar{v}+E_{z} \bar{w}+E_{t}\right)}{\alpha^{2}+E_{x}^{2}+E_{y}^{2}+E_{z}^{2}} \\
& w=\bar{w}-\frac{E_{z}\left(E_{x} \bar{u}+E_{y} \bar{v}+E_{z} \bar{w}+E_{t}\right)}{\alpha^{2}+E_{x}^{2}+E_{y}^{2}+E_{z}^{2}} .
\end{aligned}
$$

Equation (7) is a system of linear equations with about $10^{8}$ variables. Direct matrix methods such as Gauss-Jordan are computationally expensive, so we use the iterative Gauss-Seidel method [6].

Using this method, the updated $\left(u^{n+1}, v^{n+1}, w^{n+1}\right)$ is computed from $\left(\bar{u}^{n}, \bar{v}^{n}, \bar{w}^{n}\right)$ using the following iteration:

$$
u^{n+1}=\bar{u}^{n}-\frac{E_{x}\left(E_{x} \bar{u}^{n}+E_{y} \bar{v}^{n}+E_{z} \bar{w}^{n}+E_{t}\right)}{\alpha^{2}+E_{x}^{2}+E_{y}^{2}+E_{z}^{2}}
$$




$$
\begin{aligned}
& v^{n+1}=\bar{v}^{n}-\frac{E_{y}\left(E_{x} \bar{u}^{n}+E_{y} \bar{v}^{n}+E_{z} \bar{w}^{n}+E_{t}\right)}{\alpha^{2}+E_{x}^{2}+E_{y}^{2}+E_{z}^{2}} \\
& w^{n+1}=\bar{w}^{n}-\frac{E_{z}\left(E_{x} \bar{u}^{n}+E_{y} \bar{v}^{n}+E_{z} \bar{w}^{n}+E_{t}\right)}{\alpha^{2}+E_{x}^{2}+E_{y}^{2}+E_{z}^{2}} .
\end{aligned}
$$

$E_{x}, E_{y}$ and $E_{z}$ are approximations to the derivative and computed by averaging finite element differentials in two input images from intraoperative MRI, namely a reference image of the brain before deformation and a target image after a deformation, and applying Gaussian smoothing $(\sigma=0.75)$. We also approximated partial derivative of intensity over time $E_{t}$ by subtracting the reference the target image and applying Gaussian smoothing ( $\sigma=0.75)$.

We employed a hierarchical multi-resolution scheme to accommodate different scales of motions with computational efficiency. The multi-resolution speeds up the convergence of the iteration represented by (11). The resolution ratios from the original data are $\mathrm{x} 1 / 8, \mathrm{x} 1 / 4, \mathrm{x} 1 / 2$ and $\mathrm{x} 1$, by downsampling by a factor of two in the slice direction image and smoothing by a Gaussian filter kernel.

After 500 iterations in each resolution, or when convergence is observed, the estimated motion field is passed to the next resolution level using bilinear interpolation, and used as the next initial guess. $\alpha^{2}$ was 0.1 in the experiments presented in this paper.

\section{Results}

We applied the method to five sets of clinical data both for the evaluation of the method and for quantitative and qualitative evaluation of brain deformation.

In each cases 3D volume SPGR (Spoiled gradient echo recovery) with 60 slices (TR: 28.6ms, TE: 12.8ms, FOV 240x240 mm, matrix 256x128, 1 NEX, $2.5 \mathrm{~mm}$ thickness/0 spacing) were obtained before and after dura opening, once or twice during tumor resection, and after dura closure (a total of five series per case). Note that all images are acquired during surgery in the interventional MRI scanner.

\section{Volume Change Experiment}

The first part of the test covered in this section is a volume change test with images from five clinical cases: \#1 Lt. frontal mass, 47yo, male; \#2 Rt. frontal mass, 49yo, female; \#3 posterior fossa mass, 19yo male; \#4 Rt. paretal mass, 53yo male; \#5 Rt. frontal mass, 36yo, female.

The first part of the test covered in this section is a volume change test. In this test, volume change was measured by comparing the sequence of intraoperative MRI right after the opening of the dura (namely the reference data) to the image acquired in the beginning of tumor resection (namely the target data). We also measured the volume 
change after motion correction of target image. The motion correction was possible by applying the inverse deformation field (indicating displacement from the target data to the reference data) to the target image. We subtracted aligned reference images from target images in order to measure mismatched volume based on the intensity difference. From the subtraction images, we counted voxels with over a predefined intensity threshold computed from mean image intensity value of graymatter. This mean image intensity was computed from ten rectangular (10x10 pixels) regions randomly but manually selected from the reference images.

Average volume change was $10385 \mathrm{~mm}^{3}$ and motion correction reduced the volume difference to $703 \mathrm{~mm}^{3}$. The result can be also interpreted that the optical flow measurement presented can detect at least $90 \%$ of deformation from sequential intraoperative MRI.

\section{Brain Deformation Analysis}

We performed clinical feasibility tests in all of the five cases with qualitative and quantitative analysis of the deformation. Five combinations of image sets are analyzed: (1st) image scanned before dura opening vs. after dura opening, (2nd and 3rd) before dura opening vs. during tumor resection, (4th) before dura opening vs. right after dura closure. This section presents case \#4, which has illustrative findings.

Maximum cortical surface shift measured was $11 \mathrm{~mm}$ and subsurface shift around ventricle was $4 \mathrm{~mm}$. Volume change was measured by aligning the sequence of intraoperative MRI before opening of the dura to the image acquired immediately after the dura is opened.

Figure 1 shows subtraction images and deformation vector map from case \#4. Intraoperatively the cortex showed a consistent sinking, associated with cerebrospinal fluid (CSF) drainage and tumor removal. The elongation of the vectors was judged to depict the clinical finding of a consistent sinking of the cortex. The subsurface movement in the vicinity of the ventricles, away from the region of tumor resection is also detected in the right image.

Figure 2 shows sequential movements of randomly selected points along the gravity direction computed with the method (case \#1). Two sites near the cortical surface and ventricle were selected from the image taken before the craniotomy; four points $(6 \mathrm{~mm}$ apart) in each site were tracked using the volumetric optical flow. In Figure 2, the gravity-directional component of the displacement vectors shows local shifting near the cortex (maximum approximately $-8 \mathrm{~mm}$ ) and ventricle (approximately $-2 \mathrm{~mm}$ ). The global shift was observed in the tissue within approximately $10 \mathrm{~mm}$ of the surface layer. 


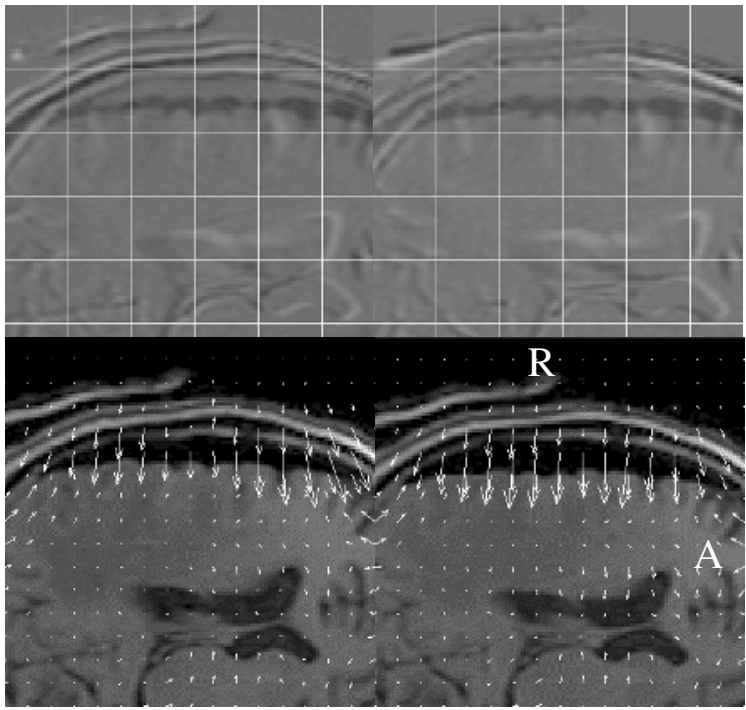

Fig. 1. Subtraction images (top left, after dura opening vs. before dura opening: top right, beginning of tumor resection vs. before dura opening) and deformation vector map (bottom left, after dura opening: bottom right, beginning of tumor resection.) from case \#4. The elongation of the vectors depicts the clinical finding of a consistent sinking of the cortex. The subsurface movement in the vicinity of the ventricles, away from the region of tumor resection is also already detected in the right image.
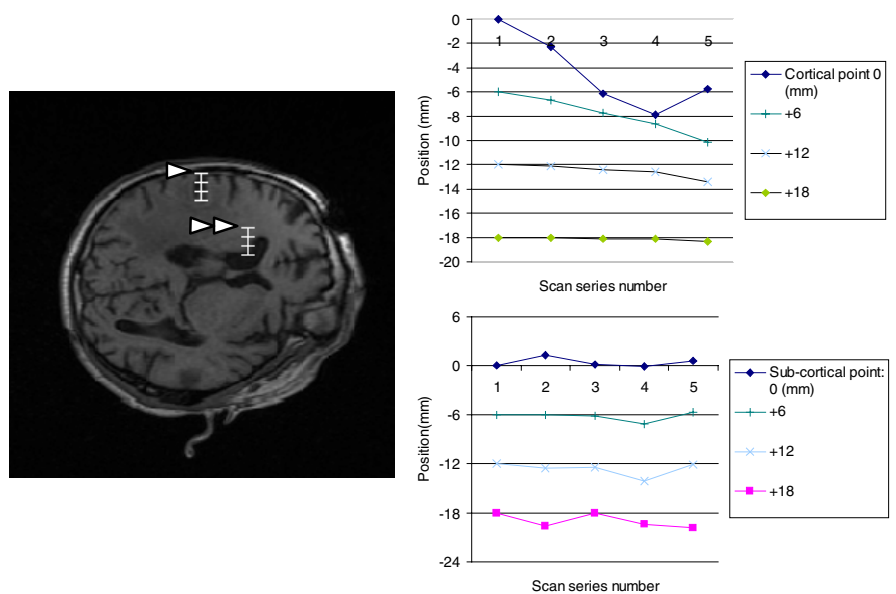

Fig. 2. The brain shift along the gravity direction by tracking four points (each $6 \mathrm{~mm}$ apart) at a cortical site (single arrow) and ventricular site (double arrow) with the volumetric optical flow (case \#1). The volumetric optical flow of $1^{\text {st }}$ and subsequent series $\left(2^{\text {nd }} \sim 5^{\text {th }}\right)$ yields the displacement of selected points during the surgery. 


\section{Discussion}

The method proposed in this paper is based on Horn and Shunck's twodimensional optical flow measurement. We employed a similar smoothness term in the optimization: the sum of squares of partial derivatives of displacements with respect to axis of coordinates. The model applies when we can assume that neighboring displacements are similar, though some studies question this assumption in real camera imagery and proposed other methods with different smoothness constraints. Alternatively, we could employ a smoothness model based on the assumption that the brain deformation field is mostly continuous.

Trobaugh and Bucholz [9] investigated intra-operative brain shift by mounting an ultrasound probe on a tracking sensor and coregistering preoperative MR/CT with intraoperative ultrasound images. The registered ultrasound images were helpful to predict intraoperative brain deformation and update mismatched preoperative images. Another publication [10] discusses three categories of depth movements. Hill [11] and Robert [12] were among the first groups to tackle measurement and quantitative analysis of intraoperative brain surface shift. Hill reported a study measuring the deformation of the dura and brain surfaces between the time of imaging and the time right after dura opening and before resection. In the surgery, the patients were first registered to preoperative images by localizing fiducial markers and performing standard patient-image registration in an image-guided surgery system. Then, they measured the location of multiple points (approx. 60) on the brain surface to compare their position with respect to those in the preoperative images. They reported mean displacements of the brain surface of 5.6-mm before tumor resection. Roberts's study used a ceiling mounted robot microscope system to measure surface displacement during surgery. They determined mean displacement of approximately 10-mm and direction of movement is presumably in the gravity direction.

Freeborough, et. al., [13] measured brain deformation to track Alzheimer's disease by nonlinear registration modified from [14]. Maurer measured the brain shift from pre- and post-operative images scanned in interventional MR setting [15] by a voxelsimilarity rigid registration by Studholme et. al. [16]. No qualitative findings about subsurface displacement were in their report except a preliminary result from threedimensional B-spline-driven deformable registration.

Both the volume change test and clinical test indicated the proposed method is capable of capturing surface and sub-surface shift of the brain. It also enables the deformable registration of pre-deformation image to post-deformation images by applying the computed motion field to the pre-deformation images; such functionality provides the means to warp intra-operative images to match pre-operative images with finer anatomical and physiological imaging capability.

\section{Acknowledgement}

NH, RK and WW were in part supported by NSF funding "Engineering Research Center for Computer-Integrated Surgical Systems and Technology" \# 9731748. AN was supported by the DFG Grant NA 359/1-1. SW was supported (in part) by grant from National Multiple Sclerosis Society. WW was supported by Whitaker Foundation. 


\section{References}

[1] J. Koivukangas, Y. Louhisalmi, J. Alakuijala, and J. Oikarinen, "Ultrasoundcontrolled neuronavigator-guided brain surgery," J Neurosurg, vol. 79, pp. 36-42, 1993.

[2] A. Kato, T. Yoshimine, T. Hayakawa, Y. Tomita, T. Ikeda, M. Mitomo, K. Harada, and H. Mogami, "A frameless, armless navigation system for computer-assisted neurosurgery," J Neurosurg, vol. 74, pp. 845-9, 1991.

[3] J. L. Barron, D. J. Fleet, and S. S. Beauchemin, "Performance of optical flow techniques," Intnational J. Comp. Vision, vol. 12, pp. 43-77, 1994.

[4] B. K. P. Horn and B. G. Schunck, "Determining optical flow," AI, vol. 17, pp. 185204, 1986.

[5] G. M. Ewing, Calculus of Variations with Applications. Mineola, NY: Dover Publications, 1985.

[6] R. Hamming, Numerical Methods for Scientists and Engineers, 2nd ed. Mineola, NY: Dover Pubns, 1987.

[7] P. A. Viola, "Alignment by Maximization of Mutual Information," in Artificial Intelligence Laboratory. Cambridge,MA: Massachusetts Institute of Technology, 1995, pp. 155.

[8] W. M. Wells III, P. Viola, H. Atsumi, S. Nakajima, and R. Kikinis, "Multi-Modal Volume Registration by Maximization of Mutual Information," Medical Image Analysis, vol. 1, pp. 35-51, 1996.

[9] J. W. Trobaugh, W. D. Richard, K. R. Smith, and R. D. Bucholz, "Frameless stereotactic ultrasonography: method and applications," Comput Med Imaging Graph, vol. 18, pp. 235-46, 1994.

[10] R. Bucholtz, D. Y. J. Trobaugh, L. McDurmont, C. Sturm, C. Baumann, J. Henderson, A. Levy, and P. Kessman, "The correction of stereotactic inaccuracy by brain shift using an intraoperative ultrasound device," presented at CVRMedMRCAS 97, Grenoble, France, 1997.

[11] D. L. Hill, C. R. Maurer, Jr., R. J. Maciunas, J. A. Barwise, J. M. Fitzpatrick, and M. Y. Wang, "Measurement of intraoperative brain surface deformation under a craniotomy," Neurosurgery, vol. 43, pp. 514-26; discussion 527-8, 1998.

[12] D. W. Roberts, A. Hartov, F. E. Kennedy, M. I. Miga, and K. D. Paulsen, "Intraoperative brain shift and deformation: a quantative analysis of cortical displacement in 28 cases," Neurosurgery, vol. 43, pp. 749-760, 1998.

[13] P. A. Freeborough and N. C. Fox, "Modeling brain deformations in Alzheimer disease by fluid registration of serial 3D MR images," J Comput Assist Tomogr, vol. 22, pp. 838-43, 1998.

[14] G. E. Chiristensen, R. D. Rabbitt, and M. I. Miller, "Deformable templates using large deformation kinematics," IEEE Trans Image Proc, vol. 5, pp. 1435-1447, 1996.

[15] C. R. Maurer, D. L. G. Hill, A. J. Martin, H. Liu, M. McCue, D. Rueckert, D. Lloret, W. A. Hall, R. E. Maxwell, D. J. Hawkes, and C. L. Truwit, "Investigation of intraoperative brain deformation using a $1.5 \mathrm{~T}$ interventional MR system: preliminary results," IEEE Trans Med Img, vol. 17, pp. 817-825, 1998.

[16] C. Studholme, D. L. G. Hill, and D. J. Hawkes, "Automated 3D registration of truncated MR and CT images of the head," presented at British medicine vision conference, 1995. 